\title{
An Interoperability Concept for Detect and Avoid and Collision Avoidance Systems: Results from a Human-in-the- Loop Simulation
}

\author{
R. Conrad Rorie ${ }^{1}$, and Lisa Fern ${ }^{2}$ \\ NASA Ames Research Center, Moffett Field, CA, 94035
}

\begin{abstract}
The integration of Unmanned Aircraft Systems (UAS) into the National Airspace System (NAS) poses a variety of technical challenges to UAS developers and aviation regulators. In response to growing demand for access to civil airspace in the United States, the Federal Aviation Administration (FAA) has produced a roadmap identifying key areas requiring further research and development. One such technical challenge is the development of a "detect and avoid" system (DAA) capable of providing a means of compliance with the "see and avoid" requirement in manned aviation. The purpose of the DAA system is to support the pilot, situated at a ground control station (GCS), in maintaining "DAA well clear" of nearby aircraft through the use of GCS displays and alerts. In addition to its primary function of aiding the pilot in maintaining DAA well clear, the DAA system must also safely interoperate with existing NAS systems and operations, such as the airspace management procedures of air traffic controllers (ATC) and Collision Avoidance (CA) systems currently in use by manned aircraft, namely the Traffic Alert and Collision Avoidance System (TCAS II). It is anticipated that many UAS architectures will integrate both a DAA system and a TCAS II. It is therefore necessary to explicitly study the integration of DAA and TCAS II alerting structures and maneuver guidance formats to ensure that pilots understand the appropriate type and urgency of their response to the various alerts. This paper presents a concept of interoperability for the two systems. The concept was developed with the goal of avoiding any negative impact on the performance level of TCAS II while retaining a DAA system that still effectively enables pilots to maintain DAA well clear. The interoperability concept described in the paper focuses primarily on facilitating the transition from a latestage DAA encounter (where a loss of DAA well clear is imminent) to a TCAS II Corrective Resolution Advisory (RA), which requires pilot compliance within five seconds of its issuance. The interoperability concept was presented to 10 participants (6 active UAS pilots and 4 active commercial pilots) in a medium-fidelity, human-in-the-loop simulation designed to stress different aspects of the DAA and TCAS II systems. Pilots' ability to maintain separation, their rate of compliance and response times using the interoperability concept are reported. Results indicated that pilots exhibited comprehension of, and appropriate prioritization within, the DAA-TCAS II combined alert structure. Pilots demonstrated a high rate of compliance with TCAS II RAs and were also seen to respond to corrective RAs within the five second requirement established for manned aircraft. The DAA system presented under test was also shown to be effective in supporting pilots' ability to maintain DAA well clear in the overwhelming majority of cases in which pilots had sufficient time to respond.
\end{abstract}

\section{Acronyms}

14CFR $=$ Title 14 Code of Federal Regulations

$A D S-B=$ Automatic Dependent Surveillance-Broadcast

ATAR

$=$ Air-to-Air RADAR

$A T C \quad=$ Air Traffic Control(ler)

${ }^{1}$ General Engineer, Aviation Systems Division, MS 262-2, AIAA Member.

${ }^{2}$ Aerospace Engineer, Aviation Systems Division, MS 262-2, AIAA Member. 


$\begin{array}{ll}C A & =\text { Collision Avoidance } \\ C P A & =\text { Closest Point of Approach } \\ D A A & =\text { Detect and Avoid } \\ D M O D & =\text { Distance Modifier } \\ F A A & =\text { Federal Aviation Administration } \\ G C S & =\text { Ground Control Station } \\ H M D & =\text { Horizontal Miss Distance } \\ I F R & =\text { Instrument Flight Rules } \\ J A D E M & =\text { Java Architecture for DAA Modeling and Extensibility } \\ \text { LoDWC } & =\text { Loss of DAA Well Clear } \\ M O P S & =\text { Minimum Operational Performance Standards } \\ N A S & =\text { National Airspace System } \\ N M & =\text { Nautical Mile } \\ N M A C & =\text { Near Mid Air Collision } \\ R A & =\text { Resolution Advisory } \\ S A R P & =\text { Science and Research Panel } \\ S C-228 & =\text { RTCA Special Committee 228 } \\ T A & =\text { Traffic Advisory } \\ T C A S I I & =\text { Traffic Alert and Collision Avoidance System } \\ T S D & =\text { Tactical Situation Display } \\ U A S & =\text { Unmanned Aircraft Systems } \\ V S C S & =\text { Vigilant Spirit Control Station } \\ Z T H R & =\text { Vertical Threshold }\end{array}$

\section{Introduction}

The integration of Unmanned Aircraft Systems (UAS) into the National Airspace System (NAS) poses a variety of technical challenges to both UAS developers and airspace regulators. In response to growing demand for access to civil airspace in the United States, the Federal Aviation Administration (FAA) has produced a roadmap that identifies key areas in need of further research and development [1]. One such technical challenge is the development of a "detect and avoid" system (DAA) capable of providing a means of compliance with the "see and avoid" requirement in manned aviation. The pilot's authority to see and avoid other traffic in order to maintain "well clear" is codified in Title 14 Code of Federal Regulations (14CFR) Part 91, sections .3, .111, .113(b), and .181(b). According to these sections, the pilot is ultimately responsible for practicing vigilance to ensure that their aircraft does not operate close enough to another aircraft so as to create a collision hazard [2]. This authority includes the right to deviate while flying Instrument Flight Rules (IFR), so long as the pilot has judged the separation between their own ship and one or more aircraft as in violation of well clear.

The see and avoid requirements under Part 91 do not provide a quantitative definition of well clear. Part 91 instead requires pilots to visually acquire the traffic and apply their own judgement as to whether or not nearby traffic poses a collision hazard. Acknowledging the need for a standardized definition of DAA well clear in the context of UAS and DAA, the FAA, with input from a Science and Research Panel (SARP), produced a definition of the concept with precise distance, time and velocity thresholds [3]. The new mathematical definition was also designed to be interoperable with existing NAS systems and procedures. In particular, the FAA and SARP ensured that the DAA well clear definition was larger than the collision avoidance volume it is meant to protect, but small enough to avoid excessive DAA well clear maneuvers from UAS aircraft, which could hinder the ability of air traffic controllers (ATC) to manage their airspace.

The newly established DAA well clear definition was adopted by RTCA Special Committee 228 (SC-228), which, with input from subject matter experts from government, industry, and academia, was responsible for developing and documenting the Phase 1 Minimum Operational Performance Standards (MOPS) for UAS DAA systems [4]. The DAA MOPS detail the requirements for the DAA system as a whole, establishing the hardware and software components that must be installed on the aircraft and in the ground control station (GCS). According to the minimum hardware requirements in the Phase 1 DAA MOPS, the UAS is required to carry active airborne surveillance (e.g., Mode S), an Automatic Dependent Surveillance-Broadcast (ADS-B) In unit, and an air-to-air RADAR (ATAR). These surveillance sources serve as input to the DAA system, which provides pilots with information critical to maintaining DAA well clear: traffic information (e.g., position, vertical trend), visual and aural DAA alerting, and DAA maneuver guidance. 
While the above (referred to as a "Class I" system) is considered the minimum equipage requirements in the Phase 1 DAA MOPS, the document optionally allows for UAS developers to install and integrate the Traffic Alert and Collision Avoidance System (TCAS II) into the aircraft and GCS alongside the DAA system. Referred to as a "Class II" system, the inclusion of TCAS II would provide pilots with protection from near midair collisions (NMAC) in the event that DAA well clear was lost. The installation of TCAS II would also ensure that pilots' Collision Avoidance maneuvers were coordinated appropriately with other TCAS II-equipped aircraft.

In order to successfully integrate these two systems, however, their distinct functions and capabilities must first be addressed.

\section{A. DAA System}

\section{DAA System Surveillance Capabilities}

In supplanting the role of visual acquisition in the see and avoid requirement, the DAA system is forced to account for all nearby traffic, regardless of the surrounding traffic's equipage. While most large aircraft (and all aircraft flying under IFR) are required to have active surveillance, smaller aircraft do not face the same requirement. Due to this discrepancy, the DAA system cannot rely solely on a transponder/receiver to detect potential threats to DAA well clear. Instead, an additional technology - such as an ATAR - is needed to detect "non-cooperative" (i.e., non-transponding) traffic within its field of regard so that the information can be displayed to the pilot on the ground [5]. The tracks detected by the ATAR will supplement the "cooperative" traffic that is detected through active surveillance and ADS-B In.

\section{DAA Alerting and Guidance}

Previous research has sought to define the GCS information requirements for pilots performing the DAA task. Specific attention has been paid to identifying the traffic display features that minimize pilot response times and the frequency and severity of losses of DAA well clear (LoDWC) [6-10]. The results of these studies informed the development of the Phase 1 DAA MOPS traffic display requirements, which specified a minimum set of information that DAA traffic displays must provide to UAS pilots. This information set includes standard traffic information (position, direction, altitude, vertical and horizontal trend) and a prescriptive multi-level DAA visual and aural alert structure.

The alerting structure specified in the Phase 1 MOPS includes two caution-level alerts and one warning-level alert. One caution-level alert is a preventive alert, which alerts the pilot to nearby traffic that is not currently predicted to lose DAA well clear but is close enough to warrant their attention. The second, higher-priority caution alert is a corrective alert, which does indicate that a loss of DAA well clear is predicted to occur and that pilot action is necessary. In the case of a corrective alert, pilots have sufficient time to contact ATC and coordinate their maneuver. The warning-level alert, conversely, is issued when a loss of DAA well clear is predicted but the pilot does not have time to coordinate with ATC before initiating an avoidance maneuver. The addition of a warninglevel alert in the DAA alerting structure was found to reduce pilot response times and minimize the rate and severity of losses of DAA well clear compared to DAA alerting structures without the alert [11].

In addition to this basic information set, the earlier research also identified the need for further conflict resolution information for UAS pilots. This additional information - referred to in the research as "DAA maneuver guidance" provides UAS pilots with explicit feedback as to which headings and altitudes (or vertical speeds) to avoid in order to prevent a loss of DAA well clear. DAA guidance is required to be "suggestive", in that it merely provides the pilot with a range of maneuver options (shown as "banding"); the decision of which precise maneuver(s) to make in response to the guidance is left to the pilot's discretion. Such information has been found to significantly improve pilot response times and loss of DAA well clear rate compared to the lack of suggestive DAA maneuver guidance [9].

Suggestive DAA maneuver guidance, however, was found to have a fundamental limitation at the point at which a loss of DAA well clear was no longer avoidable. In these cases, the DAA display was found to saturate with warning-level bands, providing the pilot with no actionable guidance information. So while maneuvering at this point in the conflict was especially important, pilots saw no viable trajectory options according to the guidance information. To account for this, researchers developed guidance to regain DAA well clear. Guidance to regain DAA well clear would engage at any point that a loss of DAA well clear could not be avoided and would highlight a narrow range of headings or altitudes/vertical speeds to achieve in order to maximize separation at the closest point of approach (CPA) between own ship and the intruder [12]. DAA maneuver guidance could then be distinguished by when in an encounter it is issued: guidance to remain DAA well clear when DAA well clear can still be maintained, and guidance to regain DAA well clear when DAA well clear is to be recovered. 


\section{B. TCAS II System}

\section{TCAS II System Surveillance Capabilities}

TCAS II was developed to provide pilots with a means of effectively maneuvering around collision avoidance threats without the aid of ATC, taking advantage of existing secondary surveillance sources (i.e., Modes A, C and S) [13]. Further, for TCAS II to provide the pilot with a vertical escape maneuver (a Resolution Advisory, or RA), the intruding aircraft must be equipped with a transponder that generates altitude reports, such as Mode $\mathrm{C}$ or $\mathrm{S}$. As such, TCAS II cannot provide protection against aircraft without a transponder.

\section{TCAS II Alerting and Guidance}

TCAS II provides pilots with standard intruder information, such as position, altitude and vertical trend, as well as a multi-level visual and aural alert structure and resolution guidance. TCAS II has two alert levels to indicate a potential collision threat: traffic advisories (TA) and RAs [14]. TAs are caution-level alerts that are intended to cue the pilot to use the TCAS II traffic display to visually acquire the alerted traffic. In doing so, the TA aids the pilot in preparing for an anticipated RA. The onset of an RA, a warning-level alert, is accompanied by an aural alert and vertical maneuver guidance. The pilot is required to immediately comply with the RA guidance, unless it is determined that compliance with the RA would jeopardize the safety of the aircraft. Different RA types require different expected pilot actions. Preventive RAs are issued to ensure that the pilot avoids certain actions, such as a climb into an intruder several hundred feet above their current altitude. Corrective RAs, conversely, require pilots to make a dedicated maneuver against an intruder in order to maximize vertical separation against a predicted collision.

The visual and aural RA alerting is accompanied by RA guidance that provides pilots with a target vertical speed or pitch angle that is predicted to result in an acceptable level of vertical separation at CPA. The typical RA guidance provides pilots with color-coded arcs on a vertical speed indicator; the red arcs indicate vertical speeds that are to be avoided, while green arcs indicate vertical speeds that are to be achieved [14]. The RA alerts and guidance remain present until own ship is clear of the conflict.

\section{Interoperability Challenges}

The fundamental differences between the DAA and TCAS II systems lead to three key areas that need to be accounted for in order to effectively present each system's features to UAS pilots.

\section{Detection of Intruders}

The DAA system is designed to detect all intruders within its available surveillance ranges. TCAS II, however, is designed to detect only cooperative traffic, providing no guidance against potential collision threats with noncooperative aircraft. The consequences of this are twofold: first, non-cooperative aircraft will not receive the additional layer of safety provided by a Collision Avoidance system; second, it is possible that the TCAS II guidance will provide an RA in the direction of non-cooperative traffic. Since non-cooperative traffic is effectively invisible to TCAS II, the TCAS II logic cannot verify that generated RAs are free from all possible conflicts. 2. Alerting Structures

The DAA and TCAS II alerting structures have potentially overlapping and contradictory alert levels. For one, both systems include caution and warning-level alerts. The caution-level alert for TCAS II is intended to assist the pilot in visual acquisition of an intruder prior to a potential RA. Without the ability to visually acquire traffic, however, UAS pilots cannot comply with this TCAS II alert. Additionally, the presence of two warning-level alerts - one from the DAA system and one from TCAS II - has the potential to confuse pilots who may expect only a single warning-level alert for traffic-related alerting systems.

Of additional note is the presence of a Preventive RA in the TCAS II alert structure, which does not necessitate pilot action, only pilot awareness (e.g., "do not climb"). This TCAS II alert goes against the philosophy used to design the DAA system alerting structure, which leveraged the FAA's definition of caution and warning-level alerts. According to Advisory Circular No. 25.1322-1, warning-level alerts should be reserved for when immediate pilot action is required, while caution-level alerts should be used when immediate pilot attention is required (with a potential need for future action) [15]. By issuing a warning-level alert for Preventive RAs, which require no corrective action by the pilot, TCAS II may be in conflict with the DAA system, which issues its own preventive alert but designates it a caution-level alert by virtue of it not requiring immediate pilot action. If unchanged, these inconsistencies could lead to pilot confusion or frustration.

\section{Maneuver Guidance}

Another key focus of integration is the display of DAA maneuver guidance immediately preceding and following the issuance of an RA. Collision threats that are sufficiently equipped will, in all but a minority of cases, lose DAA well clear before registering as an RA. Due to this, DAA maneuver guidance to regain DAA well clear (which presents a narrow range of vertical or horizontal trajectories to achieve) will already have been issued prior to the appearance of RA guidance. Since the DAA system and TCAS II are not coordinated, there is a possibility 
that the vertical guidance to regain DAA well clear will be followed by an RA in the opposite direction. The quick reversal of vertical guidance when transitioning between the two systems could violate a key assumption of the TCAS II logic, which presumes no vertical maneuvering from own ship prior to the issuance of an RA. Conceivably, the discrepancy could also encourage pilots to disregard the RA since they have already initiated a separate vertical escape maneuver in accordance with the DAA system's guidance to regain DAA well clear.

Additionally, once an RA has been issued for an intruder, the DAA maneuver guidance will likely depict all headings and altitudes as associated with a conflict (i.e., all red or amber bands). While there will be a concurrent overlay of DAA guidance to regain DAA well clear in these situation, the saturation of the banding prevents the DAA system from providing transparent maneuver guidance information for secondary threats. In the horizontal dimension, the presence of an active RA may mask conflict-free heading regions that could be an important point of reference for other nearby traffic when one is maneuvering. Since the intruder causing the horizontal band saturation is going to be resolved in the vertical dimension as a result of the RA guidance, this horizontal guidance provides no useful function. Similarly, the presence of vertical DAA maneuver guidance may confuse pilots that are attempting to respond to an active RA. Concurrent vertical DAA guidance could lead pilots to disregard (or perhaps, reverse) an RA in instances where the DAA system provides conflicting information.

\section{Interoperability Workshop}

The above challenges were addressed in a Class II system interoperability workshop consisting of members of RTCA SC-228. Members discussed the obstacles to integrating the DAA and TCAS II systems, as well as options for their mitigation. The result was an interoperability concept with two primary goals. First, the safety performance of TCAS II had to be maintained. Since the system is already certified and in widespread operation, TCAS II cannot be incorporated in a way that would diminish its effectiveness. Second, the DAA system must still be designed to limit the frequency and severity of losses of DAA well clear. In doing so, the DAA system reduces the frequency of TCAS II alerts and the subsequent need for Collision Avoidance maneuvers.

\section{Method}

\section{A. Experimental Design}

The purpose of the current study was to apply the workshop's resulting interoperability concept to a representative simulation environment in order to characterize pilot performance with the two systems. Pilots were tasked with resolving a variety of conflicts that stressed the DAA and TCAS II systems, which were designed to highlight the most vulnerable aspects of a Class II UAS system.

1. Class II System Interoperability Concept

The Class II system interoperability workshop developed a list of requirements for a combination DAA-TCAS II traffic avoidance system. Each requirement is intended to address an existing challenge regarding the systems' integration. The interoperability requirements agreed upon at the workshop are as follows:

- TCAS II Corrective RAs shall always be displayed to the pilot. Pilots shall be expected to comply with all displayed Corrective RAs provided that doing so will not jeopardize the safety of the aircraft.

- TCAS II Preventive RAs shall be presented as preventive DAA alerts to preserve the functional definitions of alert levels specified by the FAA. Any TCAS II maneuver guidance generated for a Preventive RA shall serve as input to the DAA vertical maneuver guidance to ensure pilots refrain from maneuvering in the direction of a potential Collision Avoidance threat.

- TCAS II TAs shall be suppressed entirely. Since the purpose of a TA is to attract pilot attention to a potential collision avoidance threat, the DAA system can be understood as fully replacing this function.

- Cooperative traffic shall receive both DAA and the TCAS II warning-level alerts. This is to preserve benefits associated with the DAA warning alert for pilots maintaining DAA well clear. The two different warning-level alerts, however, shall have distinct visual and aural cues, with TCAS II RAs designated as higher priority.

- Vertical guidance to regain DAA well clear shall be suppressed for cooperative traffic. This will eliminate the possibility of a TCAS II RA reversing a vertical maneuver that had been previously recommended by the regain DAA well clear guidance.

- Intruders that cause a TCAS II RA on own ship shall be removed from the calculation of horizontal guidance to remain DAA well clear. This will prevent the saturation of DAA maneuver guidance bands, and thus enable pilots to reference horizontal guidance to remain DAA well clear when responding to secondary threats with an active RA. 
- Vertical guidance to remain DAA well clear shall be suppressed during an active TCAS II RA on own ship. This will prevent pilots from receiving vertical guidance to remain DAA well clear that contradicts the guidance generated by TCAS II.

- In the event that compliance with a TCAS II RA leads to a secondary warning-level threat with a noncooperative intruder, horizontal guidance to regain DAA well clear shall be issued, regardless of whether or not a loss of well clear is avoidable. The urgency and complexity of the secondary threat requires the use of the explicit directional information that is provided by the guidance to regain DAA well clear.

\section{Participants}

Ten participants were recruited for the present study. Six of the participants were active duty UAS pilots $(M=36$ years of age), with an average 265 hours of manned flying experience and an average 230 hours of unmanned flying experience. Four commercial pilots ( $M=30$ years of age) were also recruited, with an average 2,250 hours of manned flying experience.

\section{B. Simulation Environment}

\section{Ground Control Station}

The GCS software and interfaces utilized in this experiment were provided by the Air Force Research Laboratory's Vigilant Spirit Control Station (VSCS) [16]. The software generated a single pilot display in this experiment, the tactical situation display (TSD), which provided vehicle control interfaces, as well as information regarding own ship (such as position and routing on a moving map), the experimental airspace, and nearby traffic. Traffic information included alerting and maneuver guidance from the DAA and TCAS II systems.

Pilots were able to operate their vehicle through the TSD's Holds Mode (HOLDS). HOLDS allows pilots to independently modify the aircraft's heading, altitude, and speed by entering the desired values into a keypad. The altitude could also be controlled through the use of spinners, which the pilot could click to increase or decrease the existing value in $500 \mathrm{ft}$ increments. HOLDS also included the ability to modify own ship's heading by clicking and dragging a heading bug on a compass rose surrounding the own ship icon on the moving map.

The TSD had a minimum display range of 5NM and a maximum range of 40NM. All inputs were made with a standard keyboard and mouse. VSCS provided an additional window that was not made available to the pilot, but was used by researchers to fire scripted conflicts at the own ship. At the conclusion of an encounter, the researcher would take over the control station's mouse and fire a new encounter. Upon initiating the encounter, the researcher would return the mouse to the participant.

\section{Experimental DAA System}

The Java Architecture for DAA Modeling and Extensibility (JADEM), developed at NASA Ames Research Center, generated the DAA system alerts and maneuver guidance [12]. JADEM references a configurable DAA well clear definition in order to determine if traffic within its simulated sensor ranges satisfy various alert criteria.

JADEM was configured to utilize the DAA well clear definition developed by SARP and SC-228 with the addition of a small buffer to account for the possibility of sensor uncertainty when used in the real world.

As specified by JADEM, a loss of DAA well clear occurred when an intruder was predicted to penetrate a horizontal miss distance (HMD) of 0.75NM, a vertical threshold (ZTHR) of 450ft, and a modified Tau (modTau; approximately time to CPA with a distance modifier) of $35 \mathrm{sec}$. A distance modifier (DMOD) of $0.75 \mathrm{NM}$ was also included to account for cases of slow closure rates. Threat detection utilized constant-state extrapolation. Table I shows the different DAA alert levels, as well as their associated visual and aural alerts. The DAA alerting structure was applied to both cooperative and non-cooperative aircraft alike.

Cooperative and non-cooperative traffic were detected at different simulated sensor ranges. JADEM applied a maximum lateral range of $15 \mathrm{NM}$ and a maximum vertical range of $\pm 5000 \mathrm{ft}$ to cooperative intruders to approximate the detection range requirement for TCAS II [14]. For non-cooperative intruders, JADEM applied a lateral range of $8 \mathrm{NM}$, and a field of regard with an azimuth of $\pm 110^{\circ}$ and an elevation of $\pm 20^{\circ}$ to approximate the requirement for ATAR [5].

JADEM provided guidance to remain and regain DAA well clear in addition to DAA system alerts. The suggestive

TABLE I

DAA AND TCAS II ALERT TYPES

\begin{tabular}{lll}
\hline \hline Symbol & $\begin{array}{l}\text { Name } \\
\text { (Traffic System) }\end{array}$ & Aural Alert \\
\hline 4 & $\begin{array}{l}\text { RA (TCAS II) } \\
\text { "Climb"/ } \\
\text { "Descend" }\end{array}$ \\
\hline W & $\begin{array}{l}\text { Warning Alert } \\
\text { (DAA) }\end{array}$ & $\begin{array}{l}\text { "Traffic, } \\
\text { Maneuver Now" }\end{array}$ \\
\hline Corrective Alert & "Traffic, Avoid" \\
\hline (DAA) & $\begin{array}{l}\text { Preventive Alert } \\
\text { (DAA) }\end{array}$ & $\begin{array}{l}\text { "Traffic, } \\
\text { Monitor" }\end{array}$ \\
\hline & $\begin{array}{l}\text { Target (DAA) } \\
\text { N/A }\end{array}$ \\
\hline \hline
\end{tabular}


guidance generated by JADEM provided pilots with a range of trajectory options, color-coding them according to their predicted threat level. Heading and altitude trajectories predicted by JADEM to remain DAA well clear were color-coded green, whereas those predicted to lead to a corrective or warning alert were color-coded yellow or red, respectively.

Horizontal maneuver guidance to remain DAA well clear (also referred to as "heading bands") overlaid the inner range ring on the TSD's moving map (Figure 1a). JADEM provided a full $360^{\circ}$ of horizontal banding coverage. Vertical maneuver guidance to remain DAA well clear (referred to here as "altitude bands") overlaid an altitude tape positioned to the right of the TSD's moving map (Figure 1a). JADEM provided $\pm 2000 \mathrm{ft}$ of altitude band coverage. Guidance to remain DAA well clear was updated at $1 \mathrm{~Hz}$ and was additive in the event of multiple intruders with overlapping conflict regions. As with the DAA alerts, guidance to remain DAA well clear was prioritized according to the severity of the threat.

JADEM also generated guidance to regain DAA well clear at the point at which a loss of DAA well clear could no longer be avoided. Guidance to regain DAA well clear provided pilots with target headings and altitudes that aided pilots in re-establishing DAA well clear. The guidance was displayed as a "wedge" that highlighted a limited range of trajectories (in either the horizontal or vertical dimension) predicted to maximize separation at CPA. The wedge overlaid the red altitude or heading bands, as shown in Figure 1b. A text box also appeared on the TSD to reinforce the system's recommendation (e.g., "Turn Left," "Climb").

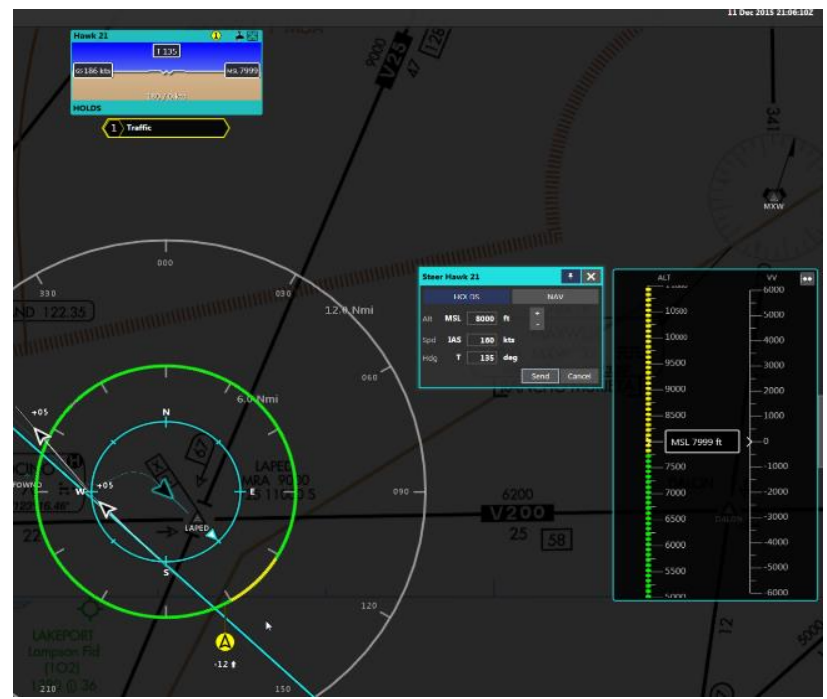

(a)

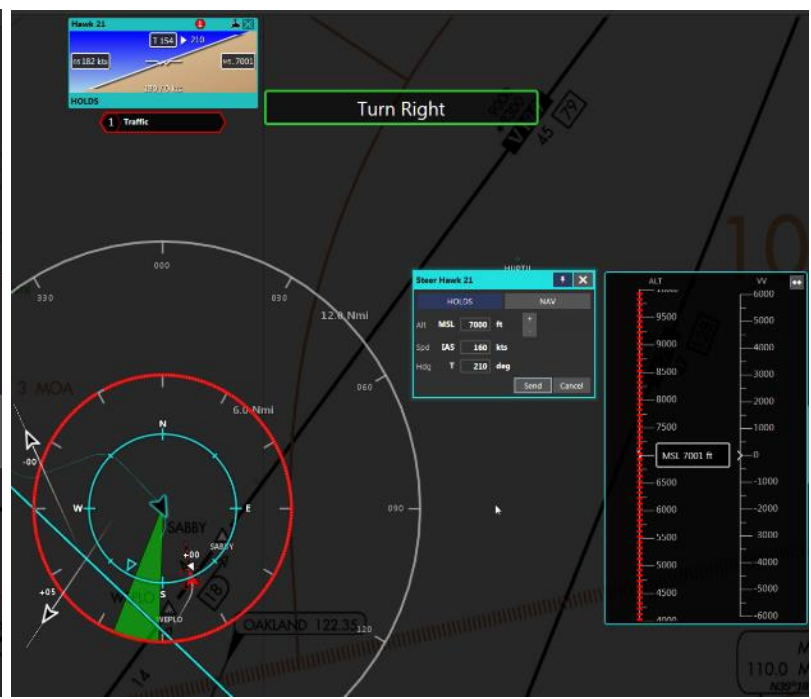

(b)

Fig. 1 Screenshots of the TSD with guidance to remain DAA well clear (a) and guidance to regain DAA well clear (b). As shown in (a), yellow bands on the inner range ring and altitude tape indicate headings and altitudes that are to be avoided. In (b), a green "wedge" and text box indicate the suggested maneuver.

\section{Experimental TCAS II System}

A TCAS II Processor, version 7.0, was utilized in this experiment. The software was integrated into NASA's simulation environment and configured to accept simulated own ship and intruder states and to output its standard alerting and guidance information to the GCS.

TCAS II utilizes altitude-based sensitivity levels (SLs). Experimental scenarios were designed to ensure the own ship remained within the altitude block of 5000-10000ft. As such, TCAS II would consistently provide SL 5 Collision Avoidance protection. At SL 5, an RA is issued when the intruder reaches a modTau of 25sec, with a ZTHR of 600ft and a DMOD of 0.55NM. In the event of an RA at SL 5, an altitude limit (ALIM) of 350ft is used to determine whether or not the RA is Preventive (i.e., requiring no pilot action) or Corrective (i.e., requiring pilot action). If vertical separation at CPA is predicted to be less than 350ft, the RA will be Corrective. Otherwise, the RA will be Preventive.

Table I above shows the visual and (typical) aural alerts associated with a Corrective RA. (Note: Preventive RAs were presented as preventive DAA alerts in the current study.) In the event of an RA, the TCAS II logic also output vertical speed guidance. The guidance provided pilots with a target vertical speed using color-coded bands overlaid on a vertical speed tape positioned on the far right side of the TSD's moving map (Figure 2). 


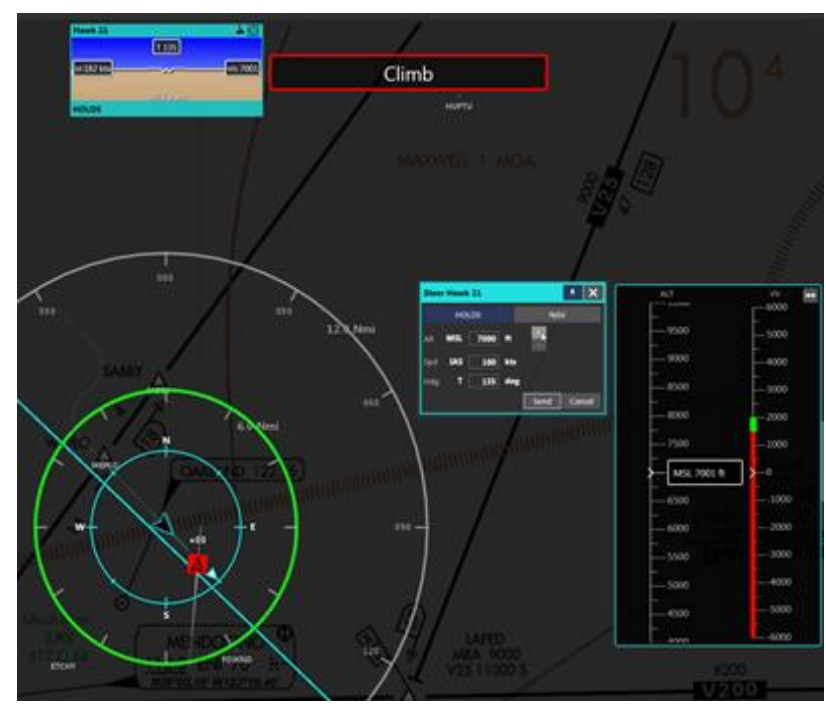

Fig. 2 Screenshot of the TSD with TCAS II RA guidance. A green band on the vertical speed tape and a text box indicate the sense of the RA.

Green arcs indicated the desired vertical speed, while red arcs indicated the vertical speeds to be avoided. A text box also appeared on the TSD when an RA was issued, indicating the sense of the RA (e.g., "Climb," "Descend"). The types of Corrective RAs possible, and their associated aural alerts and target vertical speeds, are shown in Table II. While the TCAS II logic utilized in this study was from version 7.0, the aural alerts were taken from TCAS II version 7.1 in order to provide pilots with the latest audio cues.

TABLE II TCAS II CORRECTIVE RA TYPES

\begin{tabular}{lll}
\hline \hline RA Type & Aural Alert & Required Vertical Rate \\
\hline Positive & "Climb"/"Descend" & $1500-2000$ FPM \\
Crossing & $\begin{array}{l}\text { "Crossing Climb"/"Crossing } \\
\text { Descend" }\end{array}$ & $1500-2000$ FPM \\
Maintain & $\begin{array}{l}\text { "Maintain Climb"/"Maintain } \\
\text { Descend" }\end{array}$ & $1500-4400$ FPM \\
Negative & "Reduce Climb"/"Reduce Descent" & 0 FPM
\end{tabular}

\section{Procedure}

\section{Pilot Task}

Participants operated a simulated MQ-9 (Reaper) along a straight, flat flight path. Their primary task was to respond to a variety of scripted conflicts that conformed to four general use case types. At the end of an individual encounter, participants were to recapture their default flight path and altitude. Time permitting, pilots were to coordinate their maneuvers to and from course with a researcher in the room serving as a stand-in for ATC. Unlike previous experiments by the authors, there were no secondary tasks, nor was there visible background traffic.

\section{Use Cases}

Participants were presented with scripted conflicts that were designed to address four different use cases relevant to a Class II system. Each use case included two different implementations, one that attempted to force the desired use case to occur using a last second acceleration by the intruder, and another that provided pilots with the maximum possible alert time. The latter strategy, referred to as "non-blunder" encounters because the intruder maintained a constant course, was included as a baseline to understand how pilots respond to nominal, best-case scenarios. The former case, referred to as "blunder" encounters because of the last-second acceleration, provided pilots with off-nominal scenarios, where a sudden change in the intruder triggers an immediate high-priority threat status (i.e., a loss of DAA well clear or a TCAS II RA). Since prior simulations had demonstrated pilots' effectiveness at preventing separation violations when provided with sufficient time, it was possible that pilots would never be presented with guidance to regain DAA well clear or TCAS II RA guidance without the use of blunder encounters. Conversely, without the non-blunder encounters, it would be impossible to infer pilot performance with the two systems under nominal conditions. As such, both encounter types were included in the experimental design.

As shown in Table III, Use Cases 1 and 2 involved a single threat and were designed to generate guidance to regain DAA well clear and TCAS II RAs, respectively. Use Case 1 included a single non-cooperative aircraft (i.e., 
no Mode A, C or S) that was designed to lose DAA well clear with own ship, absent pilot action. In the blunder case, the intruder began outside of the DAA well clear boundary but executed a maneuver into own ship at the point at which a loss of DAA well clear was no longer avoidable, triggering a DAA warning alert and guidance to regain DAA well clear. Since Use Case 1 utilized a non-cooperative intruder, it could not proceed to a TCAS II RA. In the Use Case 1 non-blunder scenario, pilots responded to a single non-cooperative intruder that was designed to lose DAA well clear with own ship from the start of the encounter. Pilots had the maximum look-ahead time of 55sec to respond before the loss of DAA well clear could occur.

Use Case 2 involved a single cooperative aircraft (i.e., equipped with Mode C) that was configured to trigger a TCAS II RA in the absence of a pilot response. In the blunder encounter, the intruder began DAA well clear of own ship, but executed a maneuver in own ship's direction at the TCAS II RA boundary. TCAS II requires several successive replies of the intruder's transponder to positively identify a Collision Avoidance threat. Due to this, pilots in the blunder encounters had several seconds of a DAA warning threat and guidance to regain DAA well clear before the TCAS II RA alert and guidance could be issued. In the non-blunder cases, pilots responded to a single cooperative intruder designed to penetrate the DAA well clear volume from the initialization of the encounter, with the maximum look-ahead time of 55sec. If pilots failed to avoid a loss of DAA well clear, the intruder was designed to progress to a TCAS II RA.

Use Cases 3 and 4 each involved two intruders, one cooperative and one non-cooperative. Both use cases were designed to trigger a secondary encounter with a non-cooperative intruder following pilot compliance with a TCAS II RA issued against the primary (and cooperative) intruder. In the blunder encounters for Use Cases $3 \& 4$, the cooperative intruder was initially DAA well clear of own ship, but executed a maneuver in own ship's direction at the boundary of a TCAS II RA. The vertical separation between own ship and the cooperative intruder was intentionally designed to force a TCAS II RA in the same direction as the non-cooperative intruder, which could not be detected by the TCAS II system.

In Use Case 3, the secondary intruder was positioned such that it would immediately trigger a DAA warning alert in the event of pilot compliance with TCAS II RA against the primary intruder. As specified by the interoperability concept, this forced the appearance of guidance to regain DAA well clear to aid the pilot in determining an effective evasive maneuver. In Use Case 4, the secondary intruder was positioned such that it would trigger a corrective DAA alert following compliance with the TCAS II RA. Use Cases 3 and 4 were, therefore, identical in design apart from the amount of time pilots had to respond to the secondary threat. Use Case 4 provided pilots with approximately 30 seconds additional time to respond and maintain DAA well clear than was available to pilots in Use Case 3.

In the non-blunder scenarios for Use Cases 3 and 4, the primary threat was designed to lose DAA well clear, and trigger a TCAS II RA, from the initialization of the encounter. As with Use Cases 1 and 2, pilots had the maximum look-ahead time of $55 \mathrm{sec}$ before a loss of DAA well clear could occur. The secondary intruder had a vertical offset from own ship and could only become a threat if the pilot maneuvered in its direction in response to the primary threat.

\section{Scenarios}

Eight unique encounters were generated within each of the four use cases, half as blunder encounters, and the other half as non-blunder encounters. Encounters were designed to provide a variety of approach angles (e.g., crossing, head-on) and intruder vertical rates (e.g., level, descending, climbing). All intruders had a ground speed of 205 knots. The resulting 32 encounters were divided across two scenarios, ensuring that each scenario had two blunder and two non-blunder encounters per use case.

Due to limitations in the intruder-generating software, own ship was required to be level at the initialization of each encounter, preventing the creation of any conflicts where both the own ship and the intruder were non-level. Own ship was also kept at 180 knots ground speed for each encounter.

\section{Training}

Participants were trained extensively on the GCS software following the completion of standard informed consent and demographic forms. This consisted of a short briefing regarding VSCS functionality as well as hands-on 
training highlighting the HOLDS navigation mode described above. Once participants were comfortable operating the simulated aircraft, additional training was provided that focused on the DAA and TCAS II systems.

The second briefing covered the difference between cooperative and non-cooperative intruders, namely their respective detection ranges and the inability of TCAS II to detect non-cooperative intruders. This briefing also detailed the multi-level alerting structure, which included DAA and TCAS II alerts. The visual and aural alerts associated with each alert level, as well as pilots' expected response, were covered in this section as well. Pilots were trained on DAA and TCAS II maneuver guidance last.

At the conclusion of the briefing and hands-on training sessions, pilots flew a 20-minute practice scenario to ensure they were comfortable both with the displays as well as the overall experimental procedure. At this stage of the training, researchers issued practice conflicts that included all DAA and TCAS II alert types. Researchers verified that pilots responded to traffic alerts and maneuver guidance appropriately and correctly coordinated their maneuvers when time permitted. Participants were not specifically trained on the various experimental use cases, but received extensive training on the individual components of the DAA and TCAS II systems. Pilots saw both blunder and non-blunder encounter types in the practice session and were instructed to respond only to intruders that had registered as a DAA corrective alert, a DAA warning alert, or a TCAS II Corrective RA alert.

5. Experimental Trials

Participants completed a total of four 60-minute experimental trials. Each experimental trial consisted of a traffic scenario with 16 unique encounters (four of each use case). Two different traffic scenarios were created for this study, both of which were presented twice. This resulted in 64 total encounters throughout the day, with 32 of them unique. The order of presentation for the four scenarios was counterbalanced across participants. After the first two experimental trials, pilots received an hour lunch. Following the conclusion of the experiment, pilots were provided with a post-simulation questionnaire and a verbal debrief.

\section{Metrics}

This paper reports on pilot responses to scripted conflicts under the proposed Class II system interoperability concept. The two types of encounters present in this study - blunder and non-blunder - necessitate two slightly different approaches of analysis. The non-blunder encounters are intended to gauge pilot behavior in nominal encounter scenarios. Of principal interest, therefore, is the proportion of encounters that resulted in a loss of DAA well clear and/or a TCAS II RA across all use cases. Furthermore, since pilots have sufficient time to resolve nonblunder encounters, any losses of separation should be thoroughly diagnosed. Pilots' rate of compliance with the guidance that accompanies such events (guidance to regain DAA well clear and TCAS II RA guidance) should be similarly investigated. Finally, pilot response times to a registered threat are also valuable, as they reveal on which aspects of the DAA and CA tasks pilots spend the most time. Response times in non-blunder encounters can also serve as comparison to response times observed in blunder encounters to provide insight into how pilot behavior varies by threat level.

While the proportion of encounters that result in a loss of DAA well clear and/or a TCAS II RA are highly relevant in the non-blunder encounters, these metrics offer little insight into the blunder encounters. This is due to the fact that all primary threats in the blunder encounters (i.e., the only intruder in a single-threat encounter and the cooperative intruder in a multi-threat encounter) were intentionally designed to lose DAA well clear or trigger a TCAS II RA with own ship. While the metrics serve no explanatory function for primary encounters, however, they can provide insight into pilots' ability to maintain separation from secondary threats in multi-threat encounters. Of considerably more interest in the blunder encounters is pilots' rate of compliance with instances of guidance to regain DAA well clear and TCAS II RAs. Compliance rates will provide insight into how reliable and salient pilots found these two guidance formats and the overall interoperability concept. Any instances of non-compliance should, in turn, be diagnosed in order to identify potential limitations of the interoperability concept. Lastly, pilot response times can help to characterize pilot performance on the DAA and Collision Avoidance tasks, and serve as a comparison to pilot performance in the non-blunder scenarios.

The following metrics were extrapolated using VSCS and JADEM output logs, as well as screen recordings of the TSD.

\section{A. Separation Metrics}

\section{Loss of DAA Well Clear Proportion}

The proportion of encounters where pilots failed to maintain the DAA well clear separation standard out of all threats predicted to lose DAA well clear, as detected by the DAA system (JADEM). 


\section{TCAS II Corrective RA Proportion}

The proportion of encounters where pilots experienced a TCAS II Corrective RA out of all threats designed to generate a TCAS II Corrective RA.

\section{B. Compliance Metrics}

1. Regain DAA Well Clear Guidance Compliance Proportion

The proportion of instances of guidance to regain DAA well clear with pilot compliance with the system's recommendation (i.e., left turn, right turn, climb, or descent).

2. TCAS II RA Guidance Compliance Proportion

The proportion of instances of TCAS II RA guidance with pilot compliance with the system's recommendation (i.e., climb or descent).

\section{Response Time Metrics}

1. Initial Response Time

A measure of the time it takes a pilot to initiate an input (i.e., edit) into one of the vehicle control interfaces. It is the difference between the time at which an alert first appears and the time that the relevant edit is started.

2. Initial Edit Time

A measure of the time it takes a pilot to complete the first edit into the vehicle control interfaces. It is the difference between the time at which the relevant edit is initiated and the time the first upload is sent to the aircraft.

3. Aircraft Response Time

A measure of the time it takes a pilot to upload an initial maneuver command to the aircraft in response to a traffic alert. It is the difference between the time at which an alert first appears and the time the first upload is sent to the aircraft (i.e., initial response time + initial edit time).

\section{Results}

Descriptive statistics are provided for all of the metrics described above. Each pilot saw a total of eight nonblunder encounters and eight blunder encounters for each use case. Since participants experienced a single interoperability concept, no comparative analyses were necessary. This experiment did, however, include multiple display configurations, presenting pilots with two different formats of guidance to regain DAA well clear and two different formats of guidance to remain DAA well clear. The effect of these different display configurations on pilot performance can be found in a separate paper [17].

\section{A. Non-Blunder Encounters}

Described above, the non-blunder encounters were intended to characterize performance under nominal conditions, where pilots had the full alert progression to resolve a conflict before losing DAA well clear.

\section{Separation Metrics}

Table IV lists the proportion of losses of DAA well clear against both primary and secondary intruders, as well as the proportion of TCAS II Corrective RAs, across blunder and non-blunder encounters. There were a total of three losses of DAA well clear and one instance of a TCAS II Corrective RA across all non-blunder encounters, each of which are detailed below.

One instance of a loss of DAA well clear occurred in the Use Case 2 non-blunder encounter set as a result of the pilot returning to course too soon. While the pilot failed to maintain DAA well clear when returning to course, sufficient vertical separation between the two aircraft was maintained to prevent the issuance of a TCAS II corrective RA.

The other two losses of DAA well clear, one of which also progressed to a TCAS II RA, were, at least in part, due to nuances with how the DAA system generates alerts and guidance. The first occurred in Use Case 3 when the pilot uploaded a descent to an altitude that was initially depicted as safe according to the altitude bands. The pilot failed to notice the altitude bands update to indicate that
TABLE IV SEPARATION METRICS

\begin{tabular}{lll}
\hline \hline Separation Metric & $\begin{array}{l}\text { Non-Blunder } \\
\text { Encounters }\end{array}$ & Blunder Encounters \\
\hline $\begin{array}{l}\text { Loss of DWC } \\
(\text { Primary Intruder) }\end{array}$ & $2 / 320(M=0.009)$ & $304 / 315(M=0.95)$ \\
\hline $\begin{array}{l}\text { Loss of DWC } \\
(\text { Secondary Intruder })^{\mathrm{b}}\end{array}$ & $1 / 11(M=0.09)$ & $39 / 90(M=0.43)$ \\
\hline TCAS II Corrective RA ${ }^{\mathrm{c}}$ & $1 / 240(M=0.004)$ & $235 / 235(M=1)$ \\
\hline
\end{tabular}

\footnotetext{
${ }^{a}$ Number of losses of DAA well clear (DWC) out of total number of primary intruders that registered as a corrective or warning DAA alert.

${ }^{\mathrm{b}}$ Number of losses of DWC out of total number of secondary intruders that registered as a corrective or warning DAA alert.

c Number of TCAS II Corrective RAs out of total number of primary, cooperative intruders that registered as a corrective or warning DAA alert.
} 
the recently-uploaded target altitude was no longer safe due to the presence of a secondary threat. Since the DAA alerting uses constant-state extrapolation, an alert was not generated against the secondary threat until the pilot leveled off at the new altitude, at which point it was too late to avoid a loss of DAA well clear.

The third and final instance of a loss of DAA well clear in the non-blunder encounter set occurred in Use Case 4, where it also generated a TCAS II Corrective RA. In the encounter, the pilot followed DAA maneuver guidance to pass behind the primary, cooperative intruder by initiating a large right turn. The pilot, for reasons that are unclear, had hesitated for more than 20 seconds before uploading the heading change. Because of this, the intruder progressed from a corrective DAA alert to a warning DAA alert before the own ship reached its target heading (see Figure 3). (Note that the DAA system uses current, not target, heading to determine intruder threat status.) Likely assuming the first maneuver was insufficient, the pilot subsequently uploaded a second maneuver to the aircraft. The new maneuver, a left-hand turn, drastically decreased the predicted separation between the two aircraft and quickly led to a loss of DAA well clear, which was soon followed by a TCAS II Corrective RA.

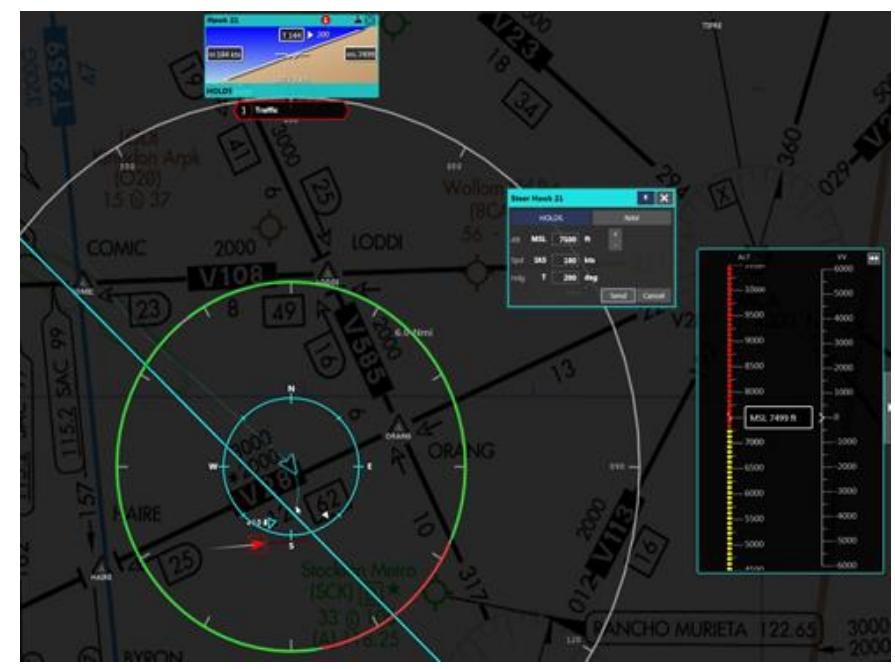

Fig. 3 Screenshot of the TSD immediately preceding a loss of DAA well clear. As shown, the aircraft is in the act of maneuvering to a new target heading, which is predicted to be conflict-free, but not yet

\section{Compliance Metrics}

Guidance to regain DAA well clear was issued three times (once for each of the above mentioned losses of DAA well clear) and pilots complied with the recommended maneuver in all three cases. TCAS II Corrective RA guidance was issued once and the pilot successfully complied.

3. Response Time Metrics

Figure 4 shows initial response time $(M=5.35 \mathrm{~s}, S E=0.65 \mathrm{~s})$, initial edit time $(M=3.30 \mathrm{~s}, S E=0.25 \mathrm{~s})$, and aircraft response time $(M=8.65 \mathrm{~s}, S E=0.66 \mathrm{~s})$, averaged across all four non-blunder use cases.

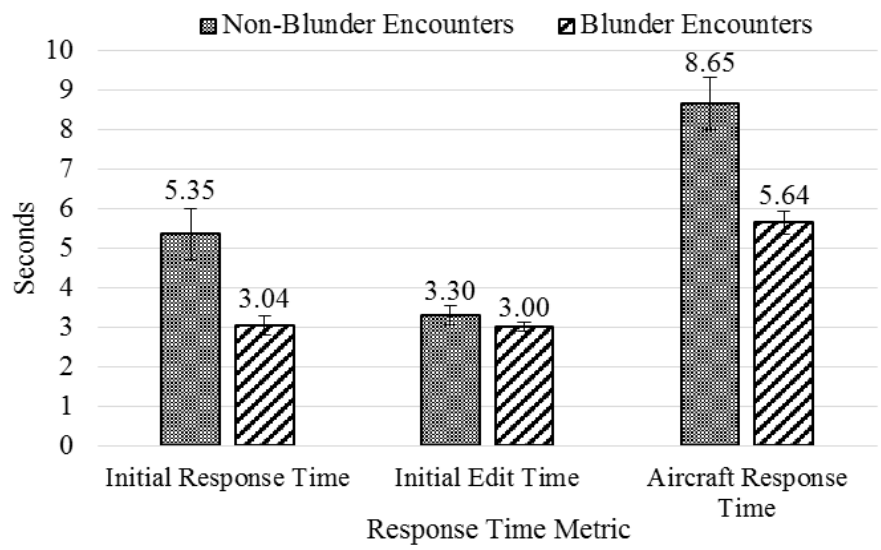

Fig. 4 Average initial response time, initial edit time, and aircraft response time for the blunder and nonblunder encounter types. 


\section{B. Blunder Encounters}

The blunder encounters were intended to stress various aspects of the DAA and TCAS II systems. To accomplish this, the primary intruder in each use case was scripted to perform a last second acceleration at the boundary of either DAA well clear or a TCAS II corrective RA.

There were five cases of missing data in the blunder encounter sets. Four of these occurred in the Use Case 3 encounter set (reducing the number of expected encounters from 80 to 76), and the fifth occurred in the Use Case 4 (dropping the number from 80 to 79). Overall, this reduced the total number of expected blunder encounters from 320 to 315 .

\section{Separation Metrics}

The primary intruder in all blunder encounters was scripted to elicit an unavoidable loss of DAA well clear or trigger a TCAS II corrective RA, regardless of pilot response. This was done in an effort to ensure the appearance of guidance to regain DAA well clear and TCAS II RA guidance. Unexpectedly, however, pilots were able to prevent a loss of DAA well clear in 11 different cases (out of 315), all of which occurred in the Use Case 1 encounter set. These corner cases were the result of pilots initiating a maneuver faster than was expected based on previous simulations (i.e., within approximately 5 seconds) against intruders with a slow closure rate. As shown in Table IV, all other encounters with a primary intruder resulted in a loss of DAA well clear. Furthermore, all primary encounters with a cooperative intruder and available data resulted in a TCAS II Corrective RA.

Unlike the primary intruder, the secondary intruder in Use Cases 3 and 4 was designed to lose DAA well clear only when the pilot complied with the TCAS II Corrective RA generated by the primary threat. As Table IV shows, pilots lost DAA well clear with the secondary intruder nearly half the time the secondary intruder registered as a threat. The likelihood of losing DAA well clear with the secondary intruder, however, was highly dependent upon the use case.

Out of the 39 losses of DAA well clear with a secondary intruder, 38 of them occurred in Use Case 3 (exactly half of the 76 Use Case 3 blunder encounters with available data). This is a result of pilots having less than 15 seconds to a loss of DAA well clear in the event that they complied with the TCAS II Corrective RA generated by the primary intruder, which, as shown below, was the majority of the time. The reason pilots failed to lose DAA well clear more than half of the time in Use Case 3, despite typically complying with the RA, was a result of pilots making multiple maneuvers against the primary intruder. While researchers presumed pilots would simply comply with the TCAS II RA vertical rate guidance, in practice, pilots often simultaneously made horizontal maneuvers. In doing so, pilots indirectly resolved conflicts against secondary intruders that were positioned head-on relative to own ship. Removing these cases, there were only four instances of pilots maintaining DAA well clear with the secondary intruder through a separate, dedicated maneuver.

The remaining loss of DAA well clear with a secondary intruder occurred in Use Case 4 when the pilot returned to course before adequate separation had been established.

\section{Compliance Metrics}

As mentioned above, there were a minority of cases where guidance to regain DAA well clear and/or TCAS II RA guidance was not generated as was expected. Nonetheless, pilots were found to comply with guidance to regain DAA well clear and TCAS II RA guidance in the vast majority of cases against primary intruders. Out of 304 cases where guidance to regain DAA well clear was issued, pilots were found to comply with the recommendation 296 times $(M=0.97)$. Of the 235 cases where TCAS II RA guidance was issued, pilots successfully complied a total of 225 times ( $M$ $=0.96$ ). A breakdown of each instance of noncompliance is shown in Table $\mathrm{V}$.

Non-compliance with guidance to regain DAA well clear against a primary intruder occurred for three different reasons: the pilot preferred a different maneuver than was suggested by the DAA system, the pilot had already maneuvered prior to receiving the guidance, or a TCAS II Corrective RA
TABLE $\mathrm{V}$

BREAKDOWN OF INSTANCES OF NON-COMPLIANCE

\begin{tabular}{|c|c|c|}
\hline Reason for Non-Compliance ${ }^{a}$ & $\begin{array}{l}\text { Guidance to Regain } \\
\text { DAA Well Clear }\end{array}$ & $\begin{array}{l}\text { TCAS II } \\
\text { Corrective RA }\end{array}$ \\
\hline $\begin{array}{l}\text { Pilot preferred different } \\
\text { maneuver than suggested by } \\
\text { guidance }\end{array}$ & 4 & 0 \\
\hline $\begin{array}{l}\text { Pilot already initiated } \\
\text { maneuver by time guidance } \\
\text { was issued }\end{array}$ & 2 & 1 \\
\hline $\begin{array}{l}\text { Pilot aware of nearby } \\
\text { secondary, non-cooperative } \\
\text { intruder (i.e., Use Case 3) }\end{array}$ & 0 & 9 \\
\hline $\begin{array}{l}\text { TCAS II issued before pilot } \\
\text { could comply with guidance to } \\
\text { regain DAA well clear }\end{array}$ & 2 & 0 \\
\hline Total & 8 & 10 \\
\hline
\end{tabular}


appeared before the pilot could respond to the guidance to regain DAA well clear.

Non-compliance with a TCAS II Corrective RA occurred most often (9 out of 10 instances) in Use Case 3, where pilots had to account for a nearby secondary, non-cooperative intruder in the same direction as the TCAS II RA guidance. Awareness of the secondary threat led pilots to avoid a vertical maneuver entirely in three of these cases, while it led pilots to make a maneuver in the sense opposite the RA in the other six cases. A final case of noncompliance with TCAS II RA guidance occurred when the pilot had already made a maneuver prior to the RA's appearance.

As described previously, pilots made far fewer dedicated maneuvers against secondary threats. This was due to pilots often having already made multiple maneuvers in response to the primary threat. As a result, data on compliance with guidance to regain DAA well clear with secondary threats is minimal. (Compliance with TCAS II RA guidance is not applicable against secondary intruders since they were non-cooperative.) Regardless, pilots were found to comply with guidance to regain DAA well clear in 20 of 21 cases where it was issued against a secondary intruder and the pilot made a dedicated maneuver against it $(M=0.95)$. The one instance of non-compliance occurred when the pilot preferred a different maneuver than was suggested by the DAA system.

It should be noted that in 40 of the 57 instances where guidance to regain DAA well clear was issued against the secondary intruder in Use Case 3, it was issued in the direction that the pilot was already flying in response to the primary threat. When including these cases, pilots complied with guidance to regain DAA well clear against a secondary intruder in 56 of 57 cases where it was issued $(M=0.98)$.

3. Response Time Metrics

Figure 4 shows initial response time $(M=3.04 \mathrm{~s}, S E=0.25 \mathrm{~s})$, initial edit time $(M=3.00 \mathrm{~s}, S E=0.12 \mathrm{~s})$, and aircraft response time ( $M=5.64 \mathrm{~s}, S E=0.30 \mathrm{~s})$ averaged across the four blunder use cases.

Response times in the blunder encounter sets with a cooperative intruder (Use Cases 2-4) can be broken down further as a function of threat type at first alert. While the primary intruder in these cases typically registered first as a DAA warning alert $(\mathrm{N}=235)$, there were a minority of cases where the intruder registered initially as a TCAS II Corrective RA $(\mathrm{N}=59)$. (This comparison was not possible in the non-blunder encounters since the intruder always registered first as a corrective DAA alert.) Figure 5 shows that pilots initiated an edit one second faster in response to a TCAS II Corrective RA $(M=1.98 \mathrm{~s}, S E=0.19 \mathrm{~s})$ than to a DAA warning alert $(M=3.00 \mathrm{~s}, S E=0.23 \mathrm{~s})$. Initial edit times were slightly less than one second faster in response to a TCAS II Corrective RA $(M=1.78 \mathrm{~s}, S E=0.09 \mathrm{~s})$ than to a DAA warning alert $(M=2.55 \mathrm{~s}, S E=0.08 \mathrm{~s})$. As a result, aircraft response times were roughly two seconds faster when pilots were responding to TCAS II Corrective RAs $(\mathrm{M}=3.62 \mathrm{~s}, \mathrm{SE}=0.17 \mathrm{~s})$ than to DAA warning alerts $(M=5.55 \mathrm{~s}, S E=0.29 \mathrm{~s})$.

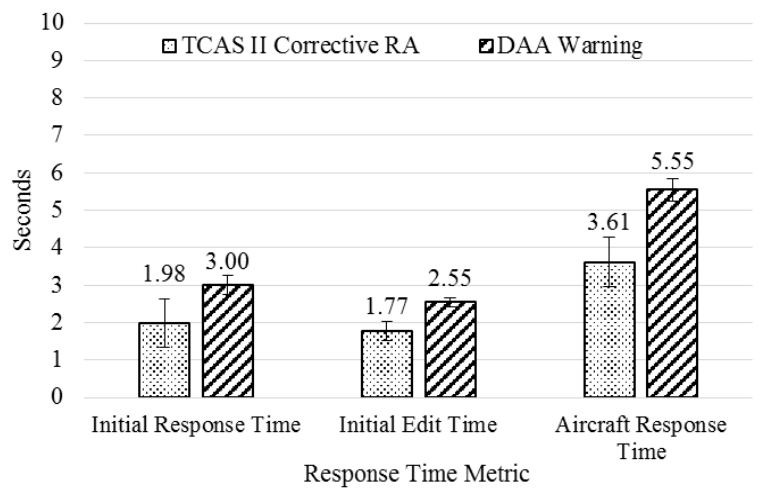

Fig. 5 Average initial response time, initial edit time, and aircraft response time by threat type at first alert.

\section{Discussion}

This paper presented a DAA-TCAS II interoperability concept to participants in a medium-fidelity simulation environment. The interoperability concept consisted of eight high-level requirements that aimed to mitigate the major incompatibilities between the two systems. Pilots were tasked with responding to four different types of use cases, each designed to stress a different part of the DAA-TCAS II system. The encounters also varied by whether or not the conflicting aircraft "blundered" into the own ship. The proposed interoperability concept was evaluated with respect to how it impacted pilots' ability to maintain separation, the frequency at which pilots complied with the various guidance types, and its associated pilot response times. 


\section{Separation Metrics}

The non-blunder encounters were designed to assess pilot performance under nominal conditions. Results indicated that pilots were overwhelmingly capable of avoiding losses of separation when given ample time under the current interoperability concept. There were only three instances of loss of DAA well clear across all non-blunder encounters, and a single TCAS II Corrective RA, which accounted for less than one percent of test encounters. As a point of comparison, the loss of DAA well clear rate in the preceding simulation by the authors ranged from 1-3\% between display configurations [10]. The slight performance improvement observed in this study can likely be attributed to the experimental protocol. Pilots had the maximum available time to resolve all threats in the nonblunder encounter set, which was not always the case in earlier studies. The present study also removed background traffic and secondary tasks, removing potential distractors and simplifying the task considerably compared to earlier experiments.

Despite high performance from participants in the present study, two of the three observed losses of separation pointed to one aspect of the DAA system that could be modified to potentially further improve pilot performance. Two losses of DAA well clear (one of which progressed to a TCAS II Corrective RA) may have been preventable if the DAA algorithm utilized own ship intent information, rather than constant-state extrapolation, when determining intruder threat status. In both cases, pilots failed to correctly evaluate the maneuver they made in response to a threat to DAA well clear. In one instance, a threat seemed to remain active despite the pilot having selected an effective new heading; in the other case, a threat appeared to be resolved but was re-triggered as soon as the aircraft leveled off at a new altitude. While pilots in the current experiment demonstrated high performance without the use of own ship intent information - hence its absence as a requirement in the Phase 1 UAS DAA MOPS - the use of intent information may further reduce the number of losses of DAA well clear attributable to pilot error.

The primary intruder in all blunder encounters was expected to lead to unavoidable losses of DAA well clear and TCAS II Corrective RAs. In spite of the design, pilots were found to maneuver the aircraft quickly enough to maintain sufficient separation with the primary intruder in 11 different encounters. Such an unexpected finding can again be attributed to the experimental protocol utilized in this study, which, as will be explained below, had a substantial impact on pilot response times.

In contrast with the primary intruder, the secondary intruder in the Use Case 3 and 4 blunder encounter sets was designed to come into conflict with own ship only if the pilot complied with the TCAS II RA generated by the primary intruder. In Use Case 3, complying with the RA was expected to lead to a DAA warning alert with the secondary threat, which the pilot would then have to resolve separately. Against expectations, pilots were observed making multiple maneuvers against the primary threat that indirectly resolved the secondary threat. Doing so allowed pilots to maintain a relatively low proportion of losses of DAA well clear (50\%) against an especially difficult multi-threat encounter.

Use Case 4 presented pilots with a similar multi-threat encounter as shown in Use Case 3, but provided pilots with an additional 30 seconds to resolve the secondary threat. The additional time reduced the number of losses of DAA well clear against the secondary intruder to a single instance, which was the result of the pilot returning to course too quickly. Pilots were again observed frequently making multiple maneuvers against the primary threat. As seen with Use Case 3, these early maneuvers often resolved the secondary threat and negated the need for a second, dedicated maneuver.

\section{Compliance Metrics}

Pilots complied with the guidance to regain DAA well clear and TCAS II RA guidance in each of the relatively few instances where they were issued in the non-blunder encounters. Compliance rates were also extremely high in the blunder encounters, where they were far more common. Compliance rates exceeded $95 \%$ for both guidance to regain DAA well clear and TCAS II RA guidance. While a high rate of compliance with guidance to regain DAA well clear is unsurprising, such a high compliance rate for TCAS II RAs was not anticipated.

The observed rates of compliance with guidance to regain DAA well clear are likely due its utility late into an encounter. Complying with guidance to regain DAA well clear is encouraged by the fact that there is no other DAA maneuver guidance available when it is issued. While pilots could ultimately decide to maneuver otherwise, the guidance to regain DAA well clear offered pilots an obvious solution. By virtue of being a function of the DAA system, guidance to regain DAA well clear is also able to account for all intruders within sensor range (which TCAS II cannot do), ensuring that it does not provide guidance that would lead to a secondary threat. While there were several cases of pilots preferring a different maneuver than what was recommended, pilots rarely had reason to noncomply.

Conversely, compliance with TCAS II was explicitly challenged by the design of the study. Half of the use cases tested in this experiment intentionally penalized pilots for compliance with a TCAS II RA by directly leading the 
own ship into a secondary threat with a non-cooperative intruder. Despite this, pilots overwhelmingly complied with the RA guidance. This is likely due, in part, to pilot training, which prioritized TCAS II and Collision Avoidance over the DAA system and the maintenance of DAA well clear. The interoperability concept reinforced this prioritization by suppressing potentially contradictory DAA information from the display. So while pilots were trained to only follow TCAS II RAs if doing so would not jeopardize the safety of the aircraft, pilots consistently erred on the side of following TCAS II. It is likely that pilots compensated for this strategy by making multiple simultaneous maneuvers against primary threats in order to de-conflict themselves with secondary intruders.

The TCAS II RA compliance rates seen here are far higher than is observed in operation. Compliance rates to Corrective RAs issued within the same altitude block flown in the current study (9000-10000ft) have been shown to be less than 50\% in live flight [18]. While precise reasons for non-compliance were not provided, it is generally presumed that in these cases pilots have visually acquired the intruding aircraft and determined that sufficient separation can be maintained without the recommended climb or descent. In relation to the Corrective RA compliance rates reported here, it is possible that pilots are less willing to disregard a TCAS II Corrective RA when they cannot acquire an intruder visually and do not have to contend with contradicting DAA alerting and guidance. The experimental design also likely contributed to the high compliance rates seen in this study. Since all primary intruders were scripted to lead to an NMAC without pilot response, there could be no realistic scenario where the pilot could independently determine that sufficient separation was going to be maintained between the two aircraft, as happens often in operation.

While compliance rates were extremely high, they were not $100 \%$. Diagnosing the instances of non-compliance revealed one major reason pilots disregarded TCAS II. Nine of the ten cases of TCAS II non-compliance in the present study were due to pilot awareness of a nearby non-cooperative intruder. A Collision Avoidance system that could account for non-cooperative traffic could prevent the system, to the extent possible, from providing Collision Avoidance maneuver guidance that conflicts with traffic detected by the DAA system. This would, in theory, help maximize pilot compliance with Collision Avoidance systems.

\section{E. Response Times}

Response times in the present study were substantially faster than had been observed in prior simulations by these authors. Aircraft response times ranged from 5.64 seconds to 8.65 seconds across the blunder and non-blunder encounters. In the most recent simulation available for comparison, aircraft response times ranged from 8.13 seconds to 18.83 seconds across display configurations and alert type [9]. This sizable difference can be largely attributed to the differences in experimental protocol already mentioned. In particular, the removal of distractions and the pairwise design of encounters primed pilots for the fastest response times possible in this environment.

Both encounter type and threat type at first alert were found to impact pilot response times. Pilots took three seconds longer to upload an initial maneuver to their aircraft in non-blunder encounters compared to blunder encounters. Nearly all of this reduction was due to faster initial response times in the blunder encounters, suggesting pilots quickly comprehended the urgency of these encounters and immediately initiated their changes in the GCS. Conversely, there was very little difference in initial edit times between the blunder and non-blunder encounters. This suggests that pilots typically knew what maneuver they were going to make by the time they started interacting with the GCS. As a result, initial edit times simply reflected a baseline level of interaction necessary to upload a heading or altitude change to the aircraft.

While response times were fast overall, pilots displayed especially quick responses when reacting to intruders that registered first as a TCAS II Corrective RA. At an average of 3.61 seconds, the aircraft response times observed here fell within the 5 seconds window that is required by the TCAS II system. This was roughly 2 seconds faster than aircraft response times to DAA warning alerts. Such a finding can be attributed to a combination of pilots' awareness of the urgency associated with a Collision Avoidance system and the benefit of directive guidance. By removing the need for pilots to determine their own resolution maneuvers, TCAS II Corrective RAs enabled slightly faster initial response times and initial edit times than alerts generated by the DAA system.

\section{Conclusion}

The current study presented a concept of interoperability for a Class II DAA-TCAS II system. Results indicated that pilots exhibited comprehension of, and appropriate prioritization within, the DAA-TCAS II combined alert structure. Pilots demonstrated a high rate of compliance with TCAS II RAs and were also seen to respond to corrective RAs within the five second requirement established for manned aircraft. The DAA system retained under

this interoperability design was also shown to be effective in supporting pilots' ability to maintain DAA well clear in the overwhelming majority of cases in which pilots had sufficient time to respond. The results of this study directly 
supported the adoption of the present interoperability concept, with minor modifications, as a part of the Phase 1 UAS DAA MOPS for Class II systems.

Future research will focus on the development of display requirements for a third class of DAA system, which will occur in Phase 2 of RTCA SC-228's UAS DAA MOPS. Class III DAA systems will utilize the next-generation Airborne Collision Avoidance System (ACAS X), the expected successor to TCAS II. A particular variant of ACAS $X$ - ACAS $X_{U}$ - is designed for vehicles with unique surveillance and performance characteristics, such as UAS [19]. ACAS $X_{U}$ is optimizable and can be configured to perform both the DAA and Collision Avoidance functions, bypassing the need for an interoperability schema as is required for Class II systems. ACAS $\mathrm{X}_{\mathrm{U}}$ will allow for total integration of CA and DAA alerting and guidance, simplifying the transition between the "remain DAA well clear" and Collision Avoidance functions.

\section{References}

[1] Federal Aviation Administration, "Integration of Civil Unmanned Aircraft Systems (UAS) in the National Airspace System (NAS) Roadmap, First Edition," Washington, DC, 2013.

[2] General Operating and Flight Rules, 14 C.F.R. § 91, Secs. .3, .111, .113(b), and .181(b), 2004.

[3] Walker, D., "FAA Position on Building Consensus Around the SARP Well-Clear Definition," RTCA Inc., SC-228 WG1 Whitepaper, Washington, DC, 2014.

[4] RTCA, "DO-365: Minimum Operational Performance Standards (MOPS) for Unmanned Aircraft Systems (UAS) Detect and Avoid (DAA) Systems," RTCA Inc., Washington, DC, 2017.

[5] RTCA, "DO-366: Minimum Operational Performance Standards (MOPS) for Air-to-Air Radar for Traffic Surveillance," RTCA, Inc., Washington, DC, 2017.

[6] Draper, D. H., Pack, J. S., Darrah, S. J., Moulton, S. N., \& Calhoun, G. L., "Human-Machine Interface Development for Common Airborne Sense and Avoid Program," Proceedings of the Human Factors and Ergonomics Society 58th Annual Meeting, Chicago, IL, 2014.

[7] Fern, L., Rorie, R.C., Pack, J., Shively, R.J., \& Draper, M., “An Evaluation of DAA Displays for Unmanned Aircraft Systems: The Effect of Information Level and Display Location on Pilot Performance," Proceedings of 15th AIAA Aviation Technology, Integration, and Operations Conference, Dallas, TX, 2015.

[8] Rorie, R. C. \& Fern, L., "The Impact of Integrated Maneuver Guidance Information on UAS Pilots Performing the Detect and Avoid Task," In Proceedings of the 59th Human Factors and Ergonomics Society Annual Meeting, Los Angeles, CA, 2015.

[9] Rorie, R. C., Fern, L., \& Shively, J., "The Impact of Suggestive Maneuver Guidance on UAS Pilots Performing the Detect and Avoid Function," Proceedings of AIAA Infotech@ Aerospace, San Diego, CA, 2016.

[10] Santiago, C., \& Mueller, E. R., "Pilot Evaluation of a UAS Detect-and-Avoid System's Effectiveness in Remaining Well Clear, In Proceedings of the Eleventh USA/Europe Air Traffic Management Research and Development Seminar (ATM2015) Lisbon, Portugal, 2015.

[11] Fern, L., "SC-228 Inclusion of DAA Warning Alert for TCAS Interoperability," NASA Technical Report (Document ID: 20160012547), Moffett Field, CA, 2016.

[12] Abramson, M., Refai, M. \& Santiago, C., "A Generic Resolution Advisor and Conflict Evaluator (GRACE) in Applications to Detect-And-Avoid (DAA) Systems of Un-manned Aircraft," Proceedings of 17th AIAA Aviation Technology, Integration, and Operations Conference, Denver, CO, 2017.

[13] US Department of Transportation, "Introduction to TCAS II Version 7.1,” Washington, DC, 2011.

[14] RTCA, "Minimum Operational Performance Standards for Traffic Alert and Collision Avoidance System II (TCAS II) Version 7.1," RTCA, Inc., Washington, DC, 2008.

[15] Department of Transportation, Advisory Circular: "Flightcrew Alerting," AC No. 25.1322-1., 2010.

[16] Feitshans, G. L., Rowe, A. J., Davis, J. E., Holland, M., \& Berger, L., "Vigilant Spirit Control Station (VSCS) - 'The Face of COUNTER'," In Proceedings of AIAA Guidance, Navigation and Control Conf. Exhibition, Honolulu, HI, 2008.

[17] Monk, K.J. \& Roberts, Z., "Maintain and Regain Well Clear: Maneuver Guidance Designs for Pilots Performing the Detectand-Avoid Task," Proceedings of the Applied Human Factors and Ergonomics International Conference, Los Angeles, CA, 2017.

[18] Olson, W. \& Olszta, J., "TCAS Operational Performance Assessment (TOPA)," Presentation at the Aviation InfoShare Meeting, Memphis TN, 2011.

[19] Manfredi, G. \& Jestin, Y, "An Introduction to ACAS Xu and the Challenges Ahead," Published in Digital Avionics Systems Conference (DASC), IEEE/AIAA 35 th , Sacramento, CA, 2016. 